\title{
Ninth annual meeting of the Italian Network for Tumor Biotherapy (NIBIT), Siena, Italy, October 19-22, 2011: New perspectives in the immunotherapy of cancer
}

\author{
Michele Maio • Hugues J. M. Nicolay • Paolo A. Ascierto • Filippo Belardelli • Roberto Camerini • \\ Mario P. Colombo · Paola Queirolo · Ruggero Ridolfi • Vincenzo Russo • Giulia Parisi • \\ Ester Fonsatti $\cdot$ Michael Papamichail $\cdot$ Giorgio Parmiani $\cdot$ on behalf of NIBIT
}

Received: 5 April 2012/ Accepted: 31 May 2012/Published online: 27 June 2012

(C) Springer-Verlag 2012

$\begin{array}{ll}\begin{array}{l}\text { Abbreviations } \\ \text { ACT } \\ \text { allo-HSCT }\end{array} & \begin{array}{l}\text { Adoptive cell therapies } \\ \text { Allogeneic hematopoietic stem cell } \\ \text { transplantation }\end{array} \\ \text { APC } & \begin{array}{l}\text { Antigen presenting cells } \\ \text { Antigen processing and presentation } \\ \text { APM }\end{array} \\ \text { machinery } \\ \text { BM } & \begin{array}{l}\text { antigen-specific cancer immunotherapeutic } \\ \text { Bone marrow }\end{array} \\ \text { CAR } & \begin{array}{l}\text { Chimeric antigen receptors } \\ \text { CIK }\end{array} \\ \end{array}$

M. Maio $(\bowtie) \cdot$ H. J. M. Nicolay · G. Parisi · E. Fonsatti

Division of Medical Oncology and Immunotherapy,

Department of Oncology, University Hospital of Siena,

Istituto Toscano Tumori, Viale Mario Bracci n. 16,

53100 Siena, Italy

e-mail: mmaio@cro.it

\section{H. J. M. Nicolay}

Cancer Bioimmunotherapy Unit, Centro di Riferimento

Oncologico, Istituto di Ricovero e Cura a Carattere Scientifico,

Aviano, Italy

\section{P. A. Ascierto}

Unit of Medical Oncology and Innovative Therapy,

Melanoma Cooperative Group, Istituto Nazionale Tumori

"Fondazione Pascale", Naples, Italy

\section{F. Belardelli}

Department of Cell Biology and Neurosciences, Istituto

Superiore di Sanità, Rome, Italy

R. Camerini

Clinical Research Unit III, Sigma Tau SpA, Pomezia, Italy

\section{P. Colombo}

Molecular Immunology Unit, Department of Experimental Oncology, Fondazione Istituto Ricovero e Cura a Carattere Scientifico, Istituto Nazionale dei Tumori, Milan, Italy

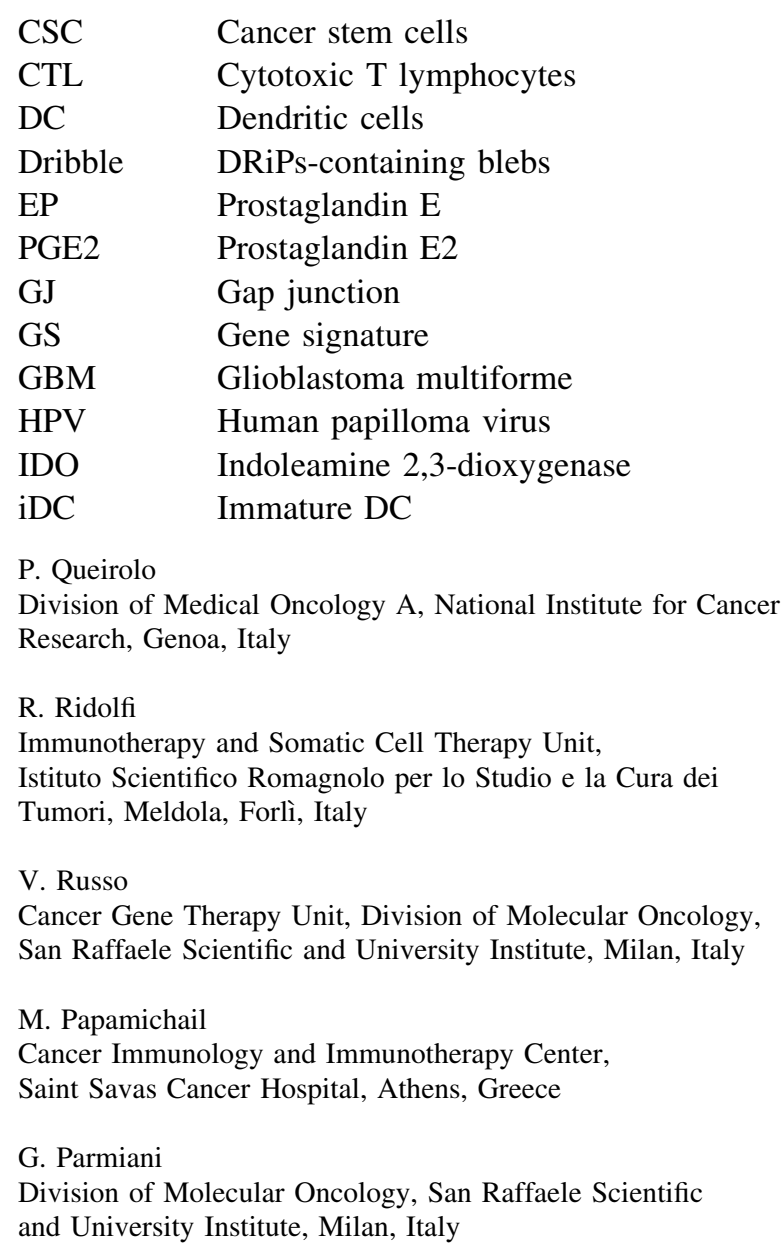

CSC

CTL

DC

Dribble DRiPs-containing blebs

EP

PGE2

GJ

GS

GBM

HPV

IDO

iDC
Cancer stem cells
Cytotoxic T lymphocytes
Dendritic cells
Prostaglandin E
Prostaglandin E2
Gap junction
Gene signature
Glioblastoma multiforme
Human papilloma virus
Indoleamine 2,3-dioxygenase
Immature DC

P. Queirolo

Division of Medical Oncology A, National Institute for Cancer

Research, Genoa, Italy

R. Ridolfi

Immunotherapy and Somatic Cell Therapy Unit,

Istituto Scientifico Romagnolo per lo Studio e la Cura dei

Tumori, Meldola, Forlì, Italy

V. Russo

Cancer Gene Therapy Unit, Division of Molecular Oncology,

San Raffaele Scientific and University Institute, Milan, Italy

M. Papamichail

Cancer Immunology and Immunotherapy Center,

Saint Savas Cancer Hospital, Athens, Greece

G. Parmiani

Division of Molecular Oncology, San Raffaele Scientific

and University Institute, Milan, Italy 


$\begin{array}{ll}\text { iNKT } & \text { Invariant NK T cells } \\ \text { LM-CRC } & \text { Liver metastases from colorectal cancer } \\ \text { mDC } & \text { Mature DC } \\ \text { mAb } & \text { Monoclonal antibody } \\ \text { MDSC } & \text { Myeloid-derived suppressor cells } \\ \text { NK } & \text { Natural Killer } \\ \text { PNT } & \text { Peroxynitrite } \\ \text { SPARC } & \text { Secreted protein acidic and rich in cysteine } \\ \text { spCD4 } & \text { CD4 + T-cell responses against short 9mer } \\ & \text { peptides } \\ \text { TAA } & \text { Tumor-associated antigens } \\ \text { TAM } & \text { Tumor-associated macrophages } \\ \text { TAPCells } & \text { Tumor APC } \\ \text { TCR } & \text { T-cell receptor } \\ \text { TIL } & \text { Tumor-infiltrating lymphocytes } \\ \text { Th } & \text { T helper } \\ \text { TLR } & \text { Toll-like receptors } \\ \text { Treg } & \text { Regulatory T cells } \\ \text { TRIMEL } & \text { Allogeneic melanoma-derived cell lysate } \\ \text { WT1 } & \text { Wilms Tumor Protein-1 } \\ \text { Young-TIL } & \text { Short-term cultured TIL }\end{array}$

\section{Introduction}

Immuno-biotherapy is a rapidly evolving field in the care of cancer patients, and it will likely continue to provide novel and effective therapeutic options in the next years. This swift evolution has been due to the in-depth understanding of basic immuno-biologic mechanisms regulating tumor-host interactions and to the rapid translation of different therapeutic agents and strategies in the clinical setting. A fundamental role in this process has been played by tight scientific interactions among highly qualified research groups; to foster these, both at basic and clinical level, different national networks focusing on immuno-biotherapy have been recently established in European countries. However, the key to further success of cancer immunobiotherapy will undoubtedly be to enlarge and strengthen collaborations at European level and to generate strong scientific and operative connections with existing nonEuropean networks interested in such a project. On these premises, the Italian Network of Tumor Biotherapy (NIBIT), established in 2004, organized its annual meeting together with that of the European Society of Cancer Immunology and Immunotherapy (ESCII) on October 19-22, 2011 in Siena, Italy. Aim of the meeting was to create a shared and structured venue to stimulate a panEuropean liaison among existing national networks to promote pre-clinical and clinical research activities in cancer immuno-biotherapy. To foster a more global interest in the field, non-academic bodies such as regulatory agencies and industry were also involved in the meeting. The meeting undoubtedly provided an appropriate environment for such a "network of national European networks".

\section{A new look at the tumor-associated antigens and targetable molecules of clinical significance}

After welcomes from authorities, the ESCII/NIBIT Meeting was opened by the excellent keynote lecture of Tobias Sjöblom (Uppsala, Sweden) providing an overview on tumor cell somatic mutations as a new source of tumor antigens to be exploited for cancer immunotherapy. He described statistical and bio-informatics tools that may help to identify mutations with a role in tumorigenesis, providing results emerged from a study on human breast and colon cancers. In summary, the data indicated that the vast majority of mutations occur in genes with very low mutation prevalence suggesting to consider more general mechanisms to avoid patient-specific approaches. Instead, Ugur Sahin (Mainz, Germany) focused his presentation on RNA antigens for targeting any type of molecular cancer alteration toward individualized cancer immunotherapy. In particular, he stressed how coding messenger RNA (mRNA) is emerging as a particularly attractive option for vaccine targeting mutated antigens. Indeed, this new technology provides with these following theoretical advantages: (1) no restriction to certain HLA alleles, (2) induction of different kind of immune responses, and (3) no integration into the genomes. Luca Sigalotti (Aviano, Italy) talked about methylationregulated tumor-associated antigens (TAA) as targets for cancer epigenetic therapeutic strategies. In this regard, he showed the great potential of selected epigenetic drugs to functionally modulate the immunogenic profile of neoplastic cells. In addition, he demonstrated that constitutive global genomic DNA hypomethylation of tumor cells associates with a significantly increased overall survival of melanoma patients, possibly through a better immune recognition of cancer cells. Overall, the data presented supported a promising clinical use of methylation-regulated TAA and epigenetic drugs in cancer immunotherapy. Pramod K. Srivastava (Farmington, USA) reported how new insights on tumor transcriptome are useful to identify tumor-specific nonsynonymous mutations that may generate putative epitopes to apply for cancer immunotherapy. He also reported on the ability of synthetic peptides to elicit effective immune response in vivo.

In his presentation, Cornelis J. Melief (Leiden, The Netherlands) provided evidence on the clinical benefit derived from the use of overlapping HPV16 synthetic long peptides comprising all potential cytotoxic $\mathrm{T}$ lymphocytes 
(CTL) and T helper (Th) epitopes for treatment of vulval intraepithelial neoplasia patients. In particular, he showed data pointing out that the success of immunotherapy is determined by the ratio of vaccine-prompted effector $\mathrm{T}$ cells over CD4 + CD25 + FoxP3 + regulatory $\mathrm{T}$ cells (Treg) and that a robust and durable response to synthetic long peptides is ascribed to the simultaneous presentation of many HLA class I and class II epitopes by dendritic cells (DC) in the absence of antigenic competition. In addition, Graham Pawelec (Tübingen, Germany) reported on CD4 + and CD8 + T-cell responses against 4 TAA (i.e., NY-ESO-1, MAGE-A3, survivin and Melan-A) by intracellular staining of selected cytokines in peripheral blood mononuclear cells of melanoma patients that had received specific different therapeutic treatments. The results indicated that (1) an unopposed pro-inflammatory CD4 + or CD8 + T-cell response to NY-ESO-1 is associated with long-term survival in stage IV melanoma, (2) only CD8 + T-cell responses to Melan-A are associated with extended survival, (3) no association with survival was seen for any responses to MAGE-A3 or survivin, and (4) responsiveness may be influenced by Cytomegalovirus infection.

Instead, Soldano Ferrone (Pittsburg, USA) focused on the endoplasmatic reticulum paralog of HSP90 (Grp94) as an interesting therapeutic target in cancer. He showed compelling evidence of the functional properties of a fully human monoclonal antibody (mAb) developed in his laboratory, with the unique specificity to recognize an extracellular epitope of Grp94 selectively expressed on neoplastic cells, able to induce apoptosis and to inhibit their proliferation in vitro. Along this line, Luigi Aurisicchio (Ariano Irpino, Italy) utilized DNA electro-genetransfer (DNA-EGT) to induce an immune response capable to generate specific antibodies aiming at neutralizing ErbB3 activity. The DNA-EGT technology was efficient in eliciting high-affinity antibodies with several biochemical and biologic activities. He provided data demonstrating that the anti-ErbB3 antibodies obtained have significant anti-tumoral properties in xenograft models and in the BALB/NeuT mice that spontaneously develop breast cancer.

\section{The microenvironment manipulation for improving the outcome of cancer immunotherapy}

In his presentation, Thomas Gajewski (Chicago, USA) reported on the molecular profiling of melanoma metastases as predictive biomarkers for response to melanoma vaccines. In particular, selected features of the tumor microenvironments such as T-cell trafficking into tumor, immune suppressive mechanisms at tumor site, tumor cell biology and susceptibility to immune-mediated killing, and complexities of the tumor stroma (vasculature and fibrosis) were analyzed. The results suggested that clinical responders to melanoma vaccines show chemokine production and an inflamed phenotype, but also high expression of negative regulators factors including PD-L1, Indoleamine 2,3dioxygenase (IDO), and FoxP3 and T-cell anergy. Along this line, Maria Pia Protti (Milan, Italy) demonstrated that the ratio of $\mathrm{Th} 2 / \mathrm{Th} 1$ infiltrating-cells in the microenvironment of pancreatic cancer has an important impact in the clinical outcome and that loco regional delivery of IFNgamma as well as anti-TNF-alpha, anti-IL-1-beta, and antithymic stromal lymphopoietin antibodies can be exploited as therapeutics to re-direct Th2-type inflammation in pancreatic cancer. In addition, Jerome Galon (Paris, France) presented compelling data indicating that the density and the immune-cell location within the tumor have a prognostic value that is superior to the TNM classification. In particular, the analysis of patients with early-stage colorectal cancer indicated the major role of CTL in predicting prognosis.

Bernard Fox (Portland, USA) discussed how to exploit autophagy to improve cancer immunotherapy. He highlighted that autophagy in tumor cells plays a critical role in cross-presentation of TAA and that autophagosomes are efficient antigen carriers for cross-priming of tumor-reactive $\mathrm{CD} 8+\mathrm{T}$ cells. On these evidences, the autophagosome-enriched vaccine named DRiPs-containing blebs (DRibble), which is derived from tumor cells after inhibition of protein degradation, was characterized. The results indicated a potent anti-tumor efficacy of the autophagosome-based DRibble vaccine whose efficacy will now be tested in clinical trials.

In her presentation, Sabina Sangaletti (Milan, Italy) reported on matricellular protein termed secreted protein acidic and rich in cysteine (SPARC). She demonstrated that SPARC distinctly regulates inflammation and collagen deposition, depending on its cellular origin. In the tumor microenvironment, SPARC might exert multiple influence but its relevance in tumor progression depends on the nature of the interaction that neoplastic cells need to establish (or not) with the surrounding microenvironment or immune cells to progress. Adriana Michielsen (Dublin, Ireland) demonstrated that the tumor microenvironment significantly influences both the maturity and function of DC in colorectal cancer patients and that the inhibition of DC maturation by the local tumor microenvironment correlates with survival of metastatic colorectal cancer patients. In addition, Alex A. Shnyra (Kansas City, USA) reported that various tumor-derived constituents may contribute to the immunosuppressive microenvironment of solid tumors including anti-inflammatory cytokines, apoptotic bodies, prostaglandin E2 (PGE2), and angiotensin II. 
In this regard, several experimental avenues were explored in the context of immunomodulation and reprogramming of tumor-associated macrophages (TAM). The results suggested that tumor-derived PGE2 may inhibit expression of Toll-like receptors (TLR) in prostaglandin E (EP)2dependent manner, whereas EP4-signaling pathway may increase expression and functional activity of TLRs in TAMs.

In his presentation, Vincenzo Russo (Milan, Italy) discussed that strategies blocking oxysterol production in combination with immunotherapy could be explored for the treatment of cancer patients. Indeed, his data demonstrated that tumor-derived oxysterols recruit bone marrow (BM)-derived neutrophils to tumors, behaving as chemotactic factors. Also, these neutrophils promote tumor growth by a pro-angiogenic activity. In conclusion, Dr. Russo pointed out that strategies blocking oxysterol production in combination with immunotherapy could be explored for the treatment of cancer patients.

\section{TAA presentation by DC and its manipulation}

DC are clearly the most potent antigen presenting cells (APC) with a crucial role in the induction of antigen-specific T-cell immune responses. In his keynote lecture, Carl G. Figdor (Nijmegen, The Netherlands) underlined issues to be addressed to optimize DC-based cancer vaccines in melanoma patients. The data obtained so far from clinical trials highlighted that vaccination studies should: (1) be performed at much earlier stages of disease, providing then a longer follow-up, (2) optimize monocyte-derived DC vaccines, (3) try to manipulate immunosuppression (Treg, myeloid-derived suppressor cells (MDSC), tumor microenvironment), (4) reach resident DC, and (5) exploit different combinations of naturally occurring DC subsets, such as blood-derived myeloid and plasmacytoid DC. On this line, Ignacio Melero (Pamplona, Spain) discussed about patients with metastatic cancer who received two cycles of four daily immunizations with monocyte-derived DC. DC were previously incubated with autologous tumor lysate and matured with IFN-alpha, TNF-alpha, and poly I:C to attain type-1 maturation. The treatment induced an increase of circulating IL-12 that correlated with the output of IL-12p70 from cultured DC from each individual and with Natural Killer (NK) activity in peripheral blood. The immunobiological effects by this combinatorial immunotherapy suggest potential activity in patients with minimal residual disease. Experimental results were also presented showing that DC can cross-present antigenic material uptaken from granulocytes in human and mouse.

The presentation of Gerold Schuler (Erlangen, Germany) focused on monocyte-derived DC matured by IL-1beta,
IL-6, TNF-alpha, PGE2, and antigen-loaded either by exogenous peptides or mRNA electroporation. In 62 melanoma patients, class I and II tumor peptide-loaded DC were administered, and immunomonitoring and long-term follow-up were performed. Of note, overall survival was markedly prolonged with a median survival of 47 months in stage IV patients. Moreover, long-term survivors (defined > 24 months) appeared to exhibit: (1) a "strong" induction of immunity in the first three months, (2) a "friendly" transcriptome pattern in pre-vaccination metastasis, and (3) eosinophilia. In patients with advanced melanoma treated with DC loaded with autologous tumor lysate, Massimo Guidoboni (Forlì, Italy) showed a signature of 24 de-regulated miRNAs (10 down- and 14 upregulated) in immature DC (iDC) of patients with better prognosis (POS) versus patients with worse prognosis (NEG), and a signature of 31 de-regulated miRNA (9 down- and 22 up-regulated) in mature DC (mDC) of POS versus NEG patients. Interestingly, 5 miRNA were common to the two signatures. These preliminary data indicate that miRNome aberrations are present at different maturation stages of DC and may account for a different response to treatment. Further investigations must be carried out to understand whether miRNA expression in iDC and $\mathrm{mDC}$ could indicate which patients will benefit of a DC vaccination and whether miRNA de-regulation is a cause or an effect of DC maturation. Cristina Martelli (Milan, Italy) discussed about Magnetic Resonance Imaging evaluation of DC vaccination protocol in a murine model of breast cancer showing that: (1) the labeling protocol did not perturb DC phenotype and functionality, permitting to visualize homing of the administered DC, (2) the use of clinically approved reagents for DC labeling will allow a faster and easier translation into clinics, (3) the vaccination results showed, at least in a preliminary study, a systemic and efficient activation of immune system.

Maria Rescigno (Milan, Italy) showed that by the use of bacteria, it is possible to induce, in both human and murine melanoma cells, the up-regulation of Connexin 43, an ubiquitous protein involved in gap junction (GJ) formation that is normally lost during melanoma progression. Bacteria-treated melanoma cells can establish functional GJ with adjacent cells, including DC. These GJ allows the transfer of antigenic pre-processed peptides to DC for T-cell activation. This Connexin 43-dependent cross-presentation pathway is more effective than standard protocols of DC loading (peptide, tumor lysates, or apoptotic bodies) for the generation of DC-based tumor vaccines that are protective in both a preventive and a therapeutic setting. This mechanism of antigen transfer has been confirmed also in human cells, and they are now ready for translation into the clinical practice. 


\section{Effectors of anti-tumor immunity}

In his keynote lecture, Pierre Coulie (Brussels, Belgium) showed data on the gene expression profiles of pre-vaccine cutaneous metastases from melanoma patients who did or did not show tumor regression following vaccination with MAGE-A3 antigen. No relevant difference between the two groups was found, but a specific inflammatory signature, quite variable between samples, and independent of the clinical evolution of the patients was observed. It comprised $\mathrm{T}$-cell and macrophage markers. The T-cell signature included activation markers, IFN-gamma target genes, and the IFN-gamma transcript itself. Results using immunohistology and microdissection on adjacent tumor sections established that this inflammatory signature colocalizes with infiltration lymphocytes in these tumors. Altogether, the data presented indicate that cutaneous melanoma metastases host various degrees of active Th1 inflammatory cells, suggesting that the immunosuppressive environment in these tumors does not result in a complete inhibition of T-cell activation.

Genetic immunization with viral vectors or plasmid DNA coding for TAA is a powerful method to evoke a protective immunity against experimental tumors, but only very limited experience exists with this vaccination approach in cancer patients. Rolf Kiessling (Stockholm, Sweden) reported on genetic Her2 vaccines made of a recombinant adenoviral vaccine based on the same kinaseinactive Her2/neu construct as a preventive or therapeutic cancer vaccine tested in preclinical models in BALB/c wt or human HER2 transgenic mice, or in a pilot clinical trial of patients with advanced breast cancer. The contribution of $\mathrm{T}$ cell, antibody responses, and of NK cells in tumor protection was tested. The results showed that in this model system, NK-mediated antibody-dependent cell-mediated cytotoxicity has a critical role in the vaccine-induced rejection of HER2-expressing tumors. Expanding on the functional role of NK cells, Lorenzo Moretta (Genoa, Italy) focused his presentation on the use of alloreactive NK cells in the successful therapy of high-risk leukemias in the T-depleted haploidentical hemopoietic stem cell (HSC) transplantation setting. The data showed that most cases analyzed the size of the alloreactive NK subset appeared to be conserved after transplantation, and persistent over time. Moreover, the cytolytic activity of the alloreactive NK subset was maintained in the recipient. In addition, a correlation between degree of NK alloreactivity and clinical outcome was clearly established.

Invariant NK T (iNKT) cells represent an intriguing subset of $\mathrm{T}$ lymphocytes that displays innate effector functions and recognizes lipid antigens presented by CD1d. Evidence suggests that iNKT cells play a sentinel role on tissue integrity and are involved in the control of pathogens, cancer, and autoimmunity. Along this line, Paolo Dellabona (Milan, Italy) discussed newly defined roles for iNKT cells in the immune surveillance of two different tumor models: (1) a mouse model of spontaneous prostate cancer, where the lack of iNKT cells results in a significantly decreased survival and enhanced tumor growth, (2) the human relevant therapeutic approach of HLA-haplodentical hematopoietic stem cell transplantation in pediatric leukemia patients, in which iNKT cell reconstitution post-transplantation correlates with leukemia control and the maintenance of the remission state.

Stefanie Gross (Erlangen, Germany) compared T-cell responses against commonly used overlapping $15 \mathrm{mer}$ peptide libraries and overlapping 9mer libraries for three vaccination antigens (i.e., MAGE-A3, Melan-A, survivin) used in clinical trials. She provided evidence that: (1) CD4 + T-cell responses against short 9mer peptides (spCD4) exist, (2) there are many different specific spCD4 T-cell epitopes, (3) spCD4 T-cell epitopes are presented in the MHC class II context, (4) spCD4 T-cell responses can be induced by vaccination, though these seem to have a relevance also in natural immune responses.

\section{Clinical studies of cancer vaccination}

In his Keynote Lecture, Pedro Romero (Lausanne, Switzerland) provided an excellent overview on new generation cancer vaccines. In particular, Romero showed how the last two decades have seen an authentic explosion of early phase clinical trials testing a large variety of cancer vaccines. Progress has also been made in the monitoring of specific T-cell responses, and efforts are underway to harmonize T-cell assays and immune biomarkers that should accompany cancer vaccine development. Finally, the advent of humanized antibodies to block specific immune checkpoints, including the FDA approval of antiCTLA-4 blockade in metastatic melanoma in March 2011, has now provided new clues for combination therapies in which therapeutic vaccines should play a central role in future immunotherapy of cancer.

The potential key role of peptide antigen characterization bears important implications for the design of novel immunotherapeutic approaches in human cancer. HansGeorg Rammensee (Tübingen, Germany) underlined that we are confronted with two layers of individuality: each human being has his or her own set of HLA molecules, with individual peptide specificities, and each tumor owns its individually accumulated set of mutations and overexpression of genes. The ultimate consequence for immunotherapy of cancer is then to identify the mutations/ alteration in the tumor, identify the new peptides presented by the HLA of the tumor, synthesize these peptides, for 
each patient individually, and finally, vaccinate the patient with these peptides, using appropriate adjuvants and conditions. Along this line, Massimo Di Nicola (Milan, Italy) discussed on the serological identification of novel lymphoma-associated antigens as candidate bio-targets for immunotherapy showing that: (1) treatment of aggressive human B-non-Hodgkin lymphoma cell lines with an antiHSP105 antibody had no direct effects on cell cycle or apoptosis but significantly reduced the tumor burden in xeno-transplanted immunodeficient mice; (2) in vivo antilymphoma activity of HSP105 engagement was associated with a significant local increase of granzyme-B + killer cells, suggesting HSP105 as a good candidate for antilymphoma treatment. Continuing the focus on the hemopoietic malignancies, Ulrich Keilhoz (Berlin, Germany) presented evidence of the immunogenicity and clinical efficacy of Wilms Tumor Protein-1 (WT1)-peptide vaccines in leukemia and solid tumors. However, further controlled clinical trials are warranted to evaluate the potential of WT1-based immunotherapy, and mechanisms of resistance are still to be thoroughly addressed.

Breast cancer is not classically thought of as an immunogenic tumor, and much less work has been done on vaccination strategies than in other cancers. In this respect, George E Peoples (San Antonio, TX, USA) presented a phase I/II dose escalation/schedule optimization clinical trial assessing the efficacy of the HER2/neu-derived E75 peptide and GM-CSF (NeuVax) in the adjuvant setting to prevent breast cancer recurrence. Similarly, he also presented a joint randomized, placebo-controlled, singleblinded phase II clinical trial of the HER2/neu-derived AE37 or GP2 peptide vaccines in breast cancer patient with any level of HER2 expression. Preliminary data showed that all three peptides appear safe and effective at raising HER2 immunity and that HER2-targeting vaccines appear effective in the adjuvant setting in decreasing breast cancer recurrences regardless of HER2 expression level.

Attention has been focused on the development of more "targeted" therapies and on predictive biomarkers to allow the identification of patients most likely to respond to a specific cancer therapy. In this context, Vincent G. Brichard (Brussels, Belgium) showed that a gene signature (GS) predictive of the clinical activity after immunization with the MAGE-A3 antigen-specific cancer immunotherapeutic (ASCI) has been identified from tumor samples taken prior immunization. In particular, in a Phase II melanoma study, overall survival was improved significantly in GS + population in comparison to the GS - population. Most of the genes identified in the GS are immune-related, suggesting for a particular immuneinfiltrated tumor environment before treatment. He reported that MAGE-A3 ASCI is currently under Phase III trial in melanoma and Non-small-cell lung carcinoma, and the identified GS under prospective evaluation.
Flavio Salazar-Onfray (Santiago, Chile) presented an original method for production of therapeutic DC-like cells named Tumor APC (TAPCells ${ }^{\circledR}$ ) using an allogeneic melanoma-derived cell lysate (TRIMEL) as activation factor and antigen provider. TAPCells-based immunotherapy-induced T-cell-mediated immune responses and improved long-term survival of stage IV patients in studies involving more than 100 individuals. Importantly, $61 \%$ of tested patients showed a Delayed Type Hypersensitivity reaction against TRIMEL, indicating the development of anti-tumor immunologic memory that correlates with prolonged patient survival. Taken together, these results indicate that TAPCells immunization resulted in two different patterns of response associated with the immunologic and clinical outcome.

Furthermore, Toos Daemen (Groningen, The Netherlands) reported on an immunotherapeutic vaccine composed of replication-defective viral replicon particles based on Semliki Forest virus (rSFV) encoding the oncoproteins E6 and E7 of human papilloma virus (HPV) type 16. In vivo data demonstrated that immunization of tumor-bearing mice with rSFVeE6, 7 replicon particles is safe and very effective in inducing HPV-specific immunity and tumor regression. The rSFV vaccine can break immune-tolerance in HPV-transgenic mice and importantly, rSFVeE6, 7 immunizations do not induce Treg while also the immune response induced is not hampered by these immunosuppressive cells. These promising preclinical studies provided the rationale to set up the first clinical phase I/II trials with a vaccine based on rSFV in patients with premalignant cervical neoplasm.

\section{Adoptive immunotherapy: where are we?}

In her keynote lecture, Chiara Bonini (Milan, Italy) focused on the genetic engineering of $\mathrm{T}$ lymphocytes for adoptive immune therapy of cancer. In particular, she illustrated that: (1) transfer of a suicide gene into donor lymphocytes and its expression therein, as a means of controlling graftversus-host disease in the context of allogeneic hematopoietic stem cell transplantation, represents the widest clinical application of T-cell-based gene transfer, (2) transfer of high-avidity T-cell receptor (TCR) genes isolated from rare tumor-specific lymphocytes into polyclonal $\mathrm{CD} 8+\mathrm{T}$ cells is an attractive strategy for targeted cancer immunotherapy, and (3) chimeric antigen receptors (CAR) usually combine the antigen binding site of a monoclonal antibody with the signal activating machinery of a $\mathrm{T}$ cell, frees antigen recognition from MHC restriction, and thus breaks one of the barriers to more widespread application of cellular therapy. Nathalie Labarriere (Nantes, France) discussed the development and evaluation of adoptive cell 
therapies (ACT) in melanoma patients using tumor-infiltrating lymphocytes (TIL) or T-cell clones. Presented results from the retrospective analysis of antigens recognized by TIL and associated with a prolonged relapse-free survival of treated patients brought to light two antigens of interest: the well-known Melan-A and a new melanoma antigen, called MELOE-1. Based on their peculiar immunogenicity and T-cell repertoire in melanoma patients, targeting of these two antigens was suggested to develop an ACT trial in melanoma patients. Robert E. Hawkins (Manchester, United Kingdom) delivered a deep overview on challenges and advantages in adoptive immunotherapy with engineered $\mathrm{T}$ cells targeted to CEA and CD19. In one hand, engineered $\mathrm{T}$ cells approach requires a large numbers of cells for each patient and special facilities/expertise. In addition, it is tailored for individual patient and is complex. On the other hand, the treatment has potential for a wide range of cancers, overcomes many checkpoints of the immune system by growing cells outside the body. Treatment of metastatic melanoma patients with adoptively transferred TIL has developed into an effective therapy. In this context, Jacob Schachter (Tel Aviv, Israel) and colleagues at the Sheba Medical Centre explored the effectiveness and toxicity of adoptive transfer of short-term cultured TIL (Young-TIL) in metastatic melanoma patients (ClinicalTrial.gov NCT00287131). Reported data showed that transfer of Young-TIL can mediate tumor regression in $42 \%$ of metastatic melanoma patients with manageable toxicity. The convincing clinical results combined with the simplification of the process may thus have a major impact on cell therapy for melanoma patients. Finally, Dario Sangiolo (Candiolo, Italy) reported on the preclinical efficacy of Cytokine-Induced Killer (CIK) cells as adoptive immunotherapy for bone and soft tissue sarcomas, with a specific focus on their anti-tumor activity against autologous tumors. This study highlighted the potential of adoptive immunotherapy with CIK cells against multiple types of currently incurable bone and soft tissue sarcomas. The original report of efficient killing versus autologous sarcoma cells addresses a crucial issue of cancer immunotherapy and leads to further in vivo preclinical studies and to the designing of adoptive immunotherapy clinical trials with CIK cells for patients with metastatic bone and soft tissue sarcomas.

\section{Why immunotherapy may not work}

In his keynote lecture, Dmitry I. Gabrilovich (Tampa, USA) showed that the anti-tumor effect of cancer immunotherapy can be improved by pharmacological regulation of the tumor microenvironment. In particular, tumor-infiltrating MDSC induce tumor cell resistance to CTL in mice by producing the free radical peroxynitrite (PNT). Along this line, he showed that pre-treatment of mouse and human tumor cells with PNT or with MDSC inhibits binding of processed peptides to tumor cell-associated MHC and, as a result, tumor cells become resistant to antigen-specific CTL. This effect was abrogated in MDSC treated with a PNT inhibitor.

The ability of cancer cells to escape from the natural or immunotherapy-induced anti-tumor immune response is often associated with alterations in the tumor cell surface expression of MHC class I antigens, and few data are available on the changes in MHC class I expression occurring during the course of cancer immunotherapy. Federico Garrido (Granada, Spain) discussed the evidence that the progression or regression of a tumor lesion in cancer patients undergoing immunotherapy could be predetermined by the molecular mechanism responsible for MHC class I molecular alterations and not by the type of immunotherapy treatment. In particular, he reported that if the alteration responsible for the changes in MHC class I expression is reversible by cytokines, the MHC class I expression will be up-regulated, the specific T-cell-mediated response will increase, and the lesion may regress. However, if the molecular defect is structural, the MHC class I expression will remain low, the escape mechanism will prevail, and the primary tumor or the metastatic lesion will progress. Furthermore, Barbara Seliger (Halle, Germany) showed that transcription factors and components of signal transduction pathways are involved in MHC class I antigen processing and presentation machinery (APM) abnormalities in human and murine tumors. She reported the association of APM deficits with reduced CTL lysis, disease progression, and patient's survival and also showed a link between functional IFN-gamma signal transduction and basal HLA class I APM expression. Finally, the investigated molecular mechanisms involved in MHC class I APM abnormalities were shown to be much more dysregulations rather than structural alterations.

In his presentation, Paolo A. Ascierto (Naples, Italy) showed in different clinical settings that: (1) high-dose Interferon-alpha $2 \mathrm{~b}$ may be effective in reducing circulating Treg in melanoma patients, (2) advanced melanoma patients non-responder to ipilimumab treatment showed a trend in increasing circulating Treg, (3) in the neoadjuvant setting of patients treated with the anti-CTLA-4 mAb ipilimumab, there seems to be a general increase of CD4 + cells (including CD4 +, CD25hi,+ FoxP3 + cells) and a decrease in MDSC.

It has been recently demonstrated that human BM cells can be treated in vitro with defined growth factors to induce the rapid generation of MDSC, hereafter defined as BM-MDSC. Vincenzo Bronte (Verona, Italy) showed that this cell population, even if displaying the structure and 
markers of promyelocytes, was, however, a distinct cell subset. In addition, he demonstrated that human BM-MDSC retain immaturity features in the presence of activated $\mathrm{T}$ lymphocytes. Finally, he presented data that BM-MDSC are equivalent to MDSC present in the blood of patients with breast cancer and patients with colorectal cancer and provided evidence that high circulating levels of MDSC in patients with advanced solid tumors are associated with radiographic disease progression and decreased survival. As far as melanoma patients, Licia Rivoltini (Milan, Italy) showed that: (1) MDSC expressing a CD11b ,$+ \mathrm{CD} 14+$, HLA-DRneg phenotype and releasing a broad array of pro-tumorigenic cyto/chemokines accumulated in the peripheral blood, (2) this accumulation is detectable as an early event, (3) MDSC conversion from normal myeloid cells could be driven by tumor exosomes preferentially accumulating in the BM.

Another hurdle discussed during this session was the immune-modulation of T-cell-mediated activity by cancer stem cells (CSC). On this issue, Cristina Maccalli (Milan, Italy) presented data using CSC isolated from glioblastoma multiforme (GBM) and their fetal bovine serum (FBS)cultured non-CSC pair lines. Results highlighted that both GBM CSC and FBS lines were weakly positive and negative for MHC-I, MHC-II, and NKG2D ligand molecules. APM were also defective in both cell types. Up-regulation of most molecules was induced by IFNs although more efficiently in FBS than in CSC. Moreover, inhibition of the proliferative activity of allogeneic T lymphocytes by GBM CSC but not by FBS cells was observed together with secretion of suppressive or pro-inflammatory cytokines by CSC versus FBS tumor cells.

The modulation of Treg in a Phase II randomized trial of cancer vaccination in stage IIB-C/III HLA-A0201 + melanoma patients was described by Chiara Castelli (Milan, Italy). Analysis of lymph nodes (LN) surgically removed after one administration of cyclophosphamide and two injections of vaccine based on the peptides Melan-A/ MART-1 (27L), Gp100 (210 M), NY-ESO-1 (165 M), and SVV-1 (97 M) suggested a trend for a reduced number of FoxP3 + cells as compared to $\mathrm{LN}$ of patients in the observation arm. Low dose of IL-2 administered after two cycles of vaccination induced a strong boost of both conventional and activated Treg. Also, Treg at the end of peptide vaccination and expanded by IL-2 included Th-1like Tregs whose functional plasticity is associated/driven to the serum availability of IL-12.

The success of immunotherapeutic strategies in patients diagnosed with high-grade glioma (HGG) is limited due to the extensive immunosuppressive environment present at tumor sites. Tina Verschuere (Leuven, Belgium) provided evidence that galectin-1 is an important player in gliomamediated immune escape since the tumor can modulate both the innate and adaptive anti-tumor immunity. Indeed, she demonstrated that local galectin-1 knock-down further boosts the anti-tumor immune response induced by immunotherapy. Thus, targeting galectin-1 may offer a novel strategy to sensitize HGG to the anti-tumor effects of immunotherapy.

Alexander Pedroza-Gonzalez (Rotterdam, The Netherlands) presented the results of a study on the impact of tumor-infiltrating Treg on tumor-specific T-cell response using ex vivo isolated cells from hepatocellular carcinoma or liver metastases from colorectal cancer (LM-CRC). The data reported suggest that Treg-mediated suppression of the local response in liver cancer might be contributing to tumor progression especially in LM-CRC.

\section{Converging strategies for immunologic monitoring}

Cancer immunotherapy represents a promising research area for the treatment of human malignancies, and the clinical translation of these therapeutic novelties is clearly improving the clinical outcome of cancer patients. Because the primary tool of immunotherapy is the immune system, studying the effects of immunotherapy by monitoring patients' immune system is crucial for immune-guiding and identifying correlates of treatment efficacy. In this session, experts and delegates of international working groups and of immune-monitoring platforms focusing on the standardization and the harmonization of immunemonitoring assays discussed how to implement such assays in order to facilitate the comparison and the integration of data across institutions and laboratories. In particular, Pedro Romero (Lausanne, Switzerland), Sylvia Janetzki (New York, USA), Cedrik Britten (Mainz, Germany), Alessandra Cesano (San Francisco, USA), Steffen Walter (Tübingen, Germany), and Cristina Maccalli (Milan, Italy) showed different ways to reach converging strategies in immunologic monitoring.

\section{Combination biological therapy}

Some regimens of chemotherapy can mediate direct cytotoxic effects on the tumor and, in addition, elicit indirect anti-tumor effects resulting from the immunogenicity of cell death. In her keynote lecture, Laurence Zitvogel (Paris, France) outlined the molecular mechanisms underlying the recognition of dying tumor cells by DC. She described four major checkpoints that dictate the immunogenicity of cell death: (1) optimal phagocytosis of chemotherapy or radiotherapy-treated tumor cells by DC requires the translocation of endoplasmic reticulum resident protein (ERp) calreticulin and disulfide isomerase ERp57 to the plasma 
membrane of dying tumor cells, (2) the chromatin-binding high mobility group box 1 protein must be released by dying tumor cells and bind to its TLR4 on DC to facilitate antigen processing of the phagocytic cargo, (3) adenosine$5^{\prime}$-triphosphate has to be released by dying cells to engage $\mathrm{P} 2 \mathrm{RX} 7$ on DC, and (4) a precise T-cell orchestration is required for the immune effectors to be able to reject the tumor.

Matteo Bellone (Milan, Italy) showed how the therapeutic efficacy of immunotherapy could increase by targeting the tumor microenvironment. Notably, he reported on the CNGRCG peptide-TNF fusion product that induces a transient up-regulation of leukocyte-endothelial cell adhesion molecules, intra-tumor release of pro-inflammatory cytokines and chemokines, and infiltration of tumorspecific effector $\mathrm{CD} 8+\mathrm{T}$ cells. He also showed that systemic treatment of tumor-bearing mice with proton pump inhibitors corrected intra-tumor acidity, favoring TIL proliferation and IFN-gamma secretion prompted by active or adoptive immunotherapy. Hence, both treatments had transient direct effects and increased the therapeutic index of T-cell-based therapies.

IDO has a vital immunosuppressive and tolerogenic function. IDO-expressing APC are found in tumor-draining $\mathrm{LN}$, and it is also expressed by tumor cells and tumor stromal cells. Thus, targeting of IDO could potentially eradicate cancer cells but also IDO + cells at the tumor site or sentinel LN. Along this line, Per thor Straten (Herlev, Denmark) showed that IDO-derived HLArestricted peptides are spontaneously recognized by host $\mathrm{CD} 8+\mathrm{T}$ cells and that such CD8 $+\mathrm{T}$ cells are capable of killing tumor cells and IDO-expressing DC. Furthermore, he demonstrated that: (1) IDO-specific T cells "unleash" and thereby support responses against tumors, (2) IDOspecific CTL may play a role as supporter $\mathrm{T}$ cells, and finally (3) targeting of IDO expression leads to decrease in Treg and up-regulation of IL-6, IL-17, and TNF-alpha. Altogether, these data provided the rationale for combining cancer vaccination against conventional TAA with IDO vaccination.

Paola Nisticò and Enrico Proietti (Rome, Italy) described a phase II randomized clinical study to prove the clinical efficacy of the administration of dacarbazine (DTIC) one day before peptide vaccination (with Melan-A and NY-ESO-1) in the prevention of melanoma relapse in clinically disease-free HLA-A2 patients. In a previous phase I study, they isolated and functionally analyzed endogenous and treatment-induced Melan-A and gp100 $\mathrm{T}$-cell clones either in patients treated with peptide vaccination alone or with DTIC one day before peptide vaccination. The combination of chemo/immunotherapy restored in Melan-A-specific T cells a high-avidity/tumorreactive T-cell clones, with a broad TCR diversity in long- surviving patients. These data suggest that the selection of immune-resistant tumor variants may be circumvented by this combination and thus prevent tumor recurrence in melanoma.

Anna Maria Di Giacomo (Siena, Italy) reported on the NIBIT-M1 trial, the first clinical trial sponsored by the NIBIT and combining the anti-CTLA- 4 mAb ipilimumab and fotemustine in metastatic melanoma patients with or without brain metastases. From July 2010 to April 2011, 86 patients were enrolled in seven Italian institutions. The preliminary results indicated that the combination is safe and feasible and shows an interesting therapeutic potential.

There is evidence that melanoma-reactive T-cell responses induced by immunotherapeutic interventions such as anti-CTLA-4 treatment or T-cell therapy with TIL can exert clinically meaningful effects. In this context, Pia Kvistborg (Amsterdam, The Netherlands) developed and validated a technology for the high-throughput detection of therapy-induced antigen-specific CD8 + T-cell reactivity and used this technology to dissect therapy-induced T-cell responses in patients treated with ipilimumab. The results showed that TIL therapy leads to a significant increase in the number of tumor-reactive T-cell responses, that melanoma specific T-cell reactivity is of low magnitude in TIL, and that T-cell responses in TIL cells predict the immune reactivity that can be seen post-therapy.

Anti-CTLA-4 mAbs are showing significant activity in different tumor types. Along this line, Luana Calabrò (Siena, Italy) reported preliminary results of a phase II, single Institution study investigating safety, clinical and immunologic efficacy of the fully human anti-CTLA- $4 \mathrm{mAb}$ tremelimumab as second-line treatment for malignant mesothelioma (MM) patients who failed a platinum-based regimen. Early results suggest that CTLA-4 blockade in MM patients is safe and shows promising clinical activity. A sizeable proportion of patients experienced clinical benefit, prolonged disease stabilization, and extension of survival. Treatment associates with major changes in activated and memory T-cell subpopulations.

\section{Conclusion}

The ESCII/NIBIT Meeting provided a highly interactive forum in which over 60 leading experts and clinical researchers in the field of cancer immunotherapy have indepth overviewed emerging topics in clinical and translational research in the area of cancer treatment. All in all, this meeting offered the opportunity to continue and strengthen the existing collaborations both nationally and internationally between basic scientists, clinical researchers, industries, and Cancer Societies to overcome obstacles in cancer care improvement at European and worldwide 
level. To do so, the meeting was concluded by a round table session, gathering experts and delegates of European Cancer Societies, to discuss about the opportunity to establish a strong Tumor Immunotherapy Network of European Networks.

Acknowledgments This work was supported in part by grants from: Istituto Superiore di Sanità - Alleanza Contro il Cancro, Associazione
Italiana per la Ricerca sul Cancro (H.J.M.N. fellow) and Istituto Toscano Tumori. A special thanks to the NIBIT members and meeting speakers for their contribution to the manuscript and the conference.

Conflict of interest The authors declare that they have no conflict of interest. 